\title{
Chapter
}

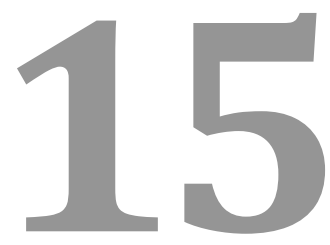

\section{APPLICATIONS OF NANOMATERIALS IN MECHANO-SENSITIVE TISSUES}

\section{M.F. Griffin and D.M. Kalaskar*}

Centre for Nanotechnology and Tissue Engineering, UCL Division of Surgery and Interventional Science, Rowland Street, University College London, NW32PF, United Kingdom 


\section{Contents}

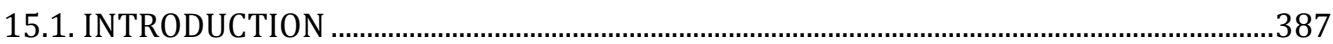

15.2. APPLICATIONS OF NANOMATERIALS IN MECHANO-SENSITIVE TISSUES...............387

15.3. NANOMATERIALS IN MECHANO-SENSITIVE TISSUES ….................................................38

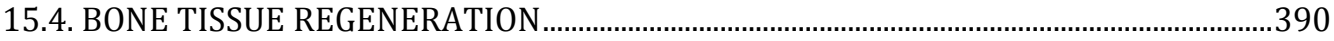

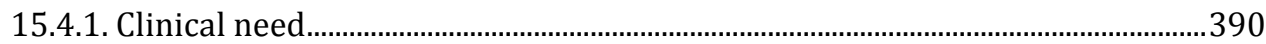

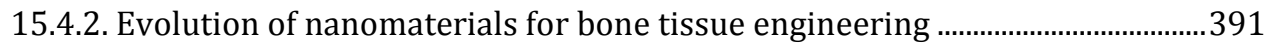

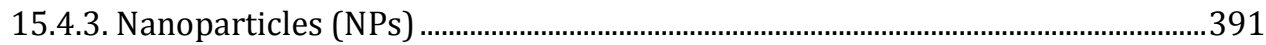

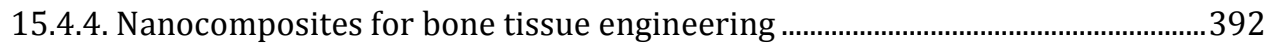

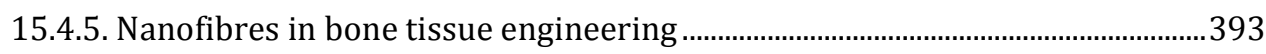

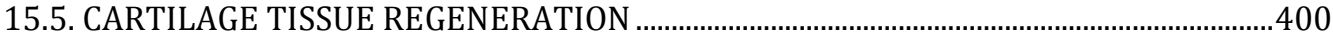

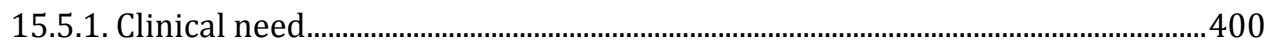

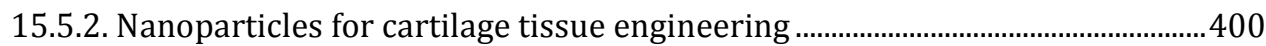

15.5.3. Nanocomposites for cartilage tissue engineering..................................................401

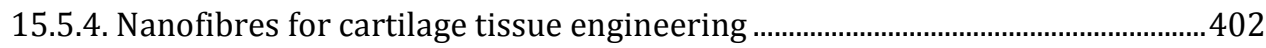

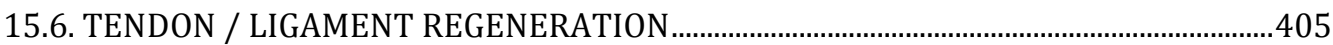

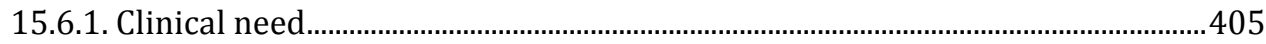

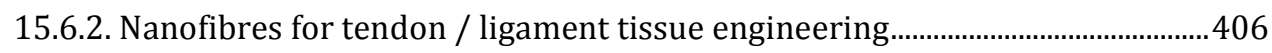

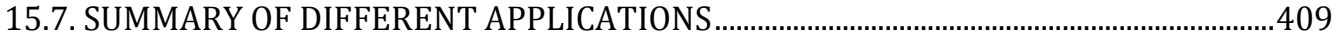

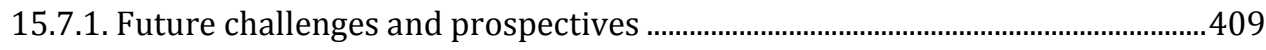

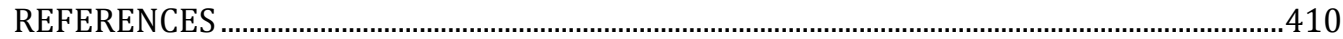




\subsection{INTRODUCTION}

Bone, cartilage and tendon defects have specific structural, chemical and biological compositions. The extracellular matrix (ECM) of these musculoskeletal tissues interacts with cells to guide tissue formation. Since the ECM of such tissues is at the nanoscale, scaffolds in development are being developed which can mimic these structure. The structural features of the scaffolds affect cell adhesion, proliferation and differentiation.

In order to repair musculoskeletal defects of bone, cartilage or tendon, synthetic materials are widely used. It is important that such materials must mimic the natural environment and provide an optimal matrix environment, biological properties including appropriate chemical cues such as growth factors and optimal mechanical properties to guide tissue regeneration. This chapter views the properties of nanomaterials for bone, cartilage and tendon regeneration. It covers the aspects of incorporating nanoparticles (NPs) with scaffolds to improve mechanical properties and the biocompatibility of polymers. The utilisation of the fabricating inert materials by electrospinning to create fibrous scaffolds that mimic the natural environment of tissues will be discussed. The design, fabrication, challenges and success of incorporating of growth factors, genetic cues and drugs to enhance bone, cartilage and tendon formation will also be evaluated. An overview of the future challenges and directions of nanomaterials for musculoskeletal tissues will be provided.

\subsection{APPLICATIONS OF NANOMATERIALS IN MECHANO-SENSITIVE TISSUES}

Tissue engineering and regenerative medicine is a rapidly growing area and aims to find alternatives for transplanting tissues to restore damaged or nonfunctioning tissues [1,2]. The musculoskeletal tissues bone, cartilage and ligament / tendon are highly structured composite tissues consisting of fibres within a matrix [1,2]. Musculoskeletal defects often heal after anatomical correction and correct wound management $[1,2]$. However, when the distance is too large to bridge, current surgical techniques to restore such defects are inadequate, which leads to poor outcomes for the patient [1,2]. With most bone lesions, artificial biomaterials are used to fill the gaps aiming to cause bone repair and regeneration [1,2]. To repair ligament and tendon injuries, the injured tissues are aligned and bridged with a biomaterial support. With cartilaginous defects natural or synthetic materials aims to support the tissue regeneration and restoration of function [3]. However, despite the improvements in surgical techniques and biomaterial characteristics such restorative strategies often do not lead to full repair of the damaged 
musculoskeletal tissue. Due to the inadequate surgical treatment options available there is a large clinical demand to create novel materials that have improved mechanical properties, enhanced material-tissue interaction that mimic the natural environment and ability to support tissue growth and organ formation $[4,5]$.

The main strategy for tissue engineering research is restoring tissue by implanting scaffold with cells. One of the major challenges in regenerative medicine is to manufacture suitable scaffolds, which elicit the correct response from the local cells to form functional organ replacements [6,7]. ECM plays an important role in these interactions with the cell surface receptors, growth factors, which provide instrumental cues for cell survival and cell fate $[6,7]$. Scientific research carried out in last few decades has shown that the interaction of cells with nanotopographical features is important in engineering complex tissues, as cells respond to various topographical features at nanoscale such as nanopores, ridges, grooves and fibres within their environment [6,7].

Over the last decade, the use of nanotechnology has further accelerated the growth in regenerative medicine. Nanomaterials comprises of scaffolds with structural dimensions of less than $100 \mathrm{~nm}[8,9]$. As biological tissues contain components, which are of this scale including collagen fibrils and hydroxyapatite (HA) crystals, cartilage and bone could also be referred to as bionano composite materials [8,9]. Furthermore, as the cell-cell interaction is at the nanoscale level, using nanotechnology provides a significant advantage in the ability to mimic the native tissue or organ $[8,9]$.

Nanocomposite scaffolds containing biocompatible polymers and bioactive NPs have been investigated with particular interest for bone tissue engineering due to their optimal mechanical properties and suitable biocompatibility $[10,11]$. Among the scaffolds evaluated, there are specific groups of nanomaterials tested for regenerative medicine including NPs, carbon nanotubes, nanofibres and composite nanomaterials. Among these electrospun nanofibres meshes have been used extensively due to their ability to mimic the collagen and elastin of the natural ECM $[10,11]$. In addition, bioactive factors can be loaded directly into the nanofibres produced via electrospining. The bioactive molecules can be released at a controlled rate and used to stimulate the proliferation and differentiation of adhered cells. One of the biggest challenges is how to preserve the bioactivity of the growth factors within the nanoscaffolds [12,13]. Several techniques have been utilised to absorb bioactive molecules within scaffolds for musculoskeletal applications [12,13].

This chapter provides the most updated overview on various types of nanomaterials, which are being investigated and are in use for mechanosensitive tissues including bone, cartilage, ligament and tendon regeneration. Special focus is given to the use of NPs, nanofibres, nanotubes and composite nanomaterials. The success and challenges of the incorporation of drugs, 
proteins, growth factors with nanomaterials will also be discussed to regenerate musculoskeletal tissues.

\subsection{NANOMATERIALS IN MECHANO-SENSITIVE TISSUES}

Nanotechnology has become a major frontier in advancing the field of musculoskeletal tissue engineering including the regeneration of bone, cartilage, tendon and ligament. The structure of bone, cartilage and ligament / tendon is very similar, they all have a hierarchical structure and in simple terms consists of a network of collagen fibrils with diameters of approximately $100 \mathrm{~nm}$, which is encased in a tissue specific matrix $[10,11]$. It is this structural design and the interactions of the fibres with the matrix that defines the mechanical and biological characteristics of each tissue type. In general the repair of a skeletal defect requires the insertion of an autologous material or synthetic material to bridge the gap, which may remain or resorb with time as the defect heals. Several materials have been investigated over the years for musculoskeletal regeneration but one of the main factors preventing the development of successful materials is the failure to mimic the natural hierarchy of the native tissue structure $[10,11]$. Thus, it is not surprising that many groups have tried to utilise nanotechnology to incorporate nano-sized components into the materials to improve the success of the regeneration of tissue $[10,11]$. There are two main approaches by which this has been researched to date. Firstly, the native nanostructured hierarchy of the tissues can be mimicked to improve the invasion of appropriate cells to create the required tissue. Secondly, nanosized components can be incorporated to ensure the mechanical properties are like those of native tissue. In the field of nanotechnology, there are four main types of nanomaterials that can be nanofibres, nanotubes and nano-structured composites, of which will be detailed below (Figure 1). 


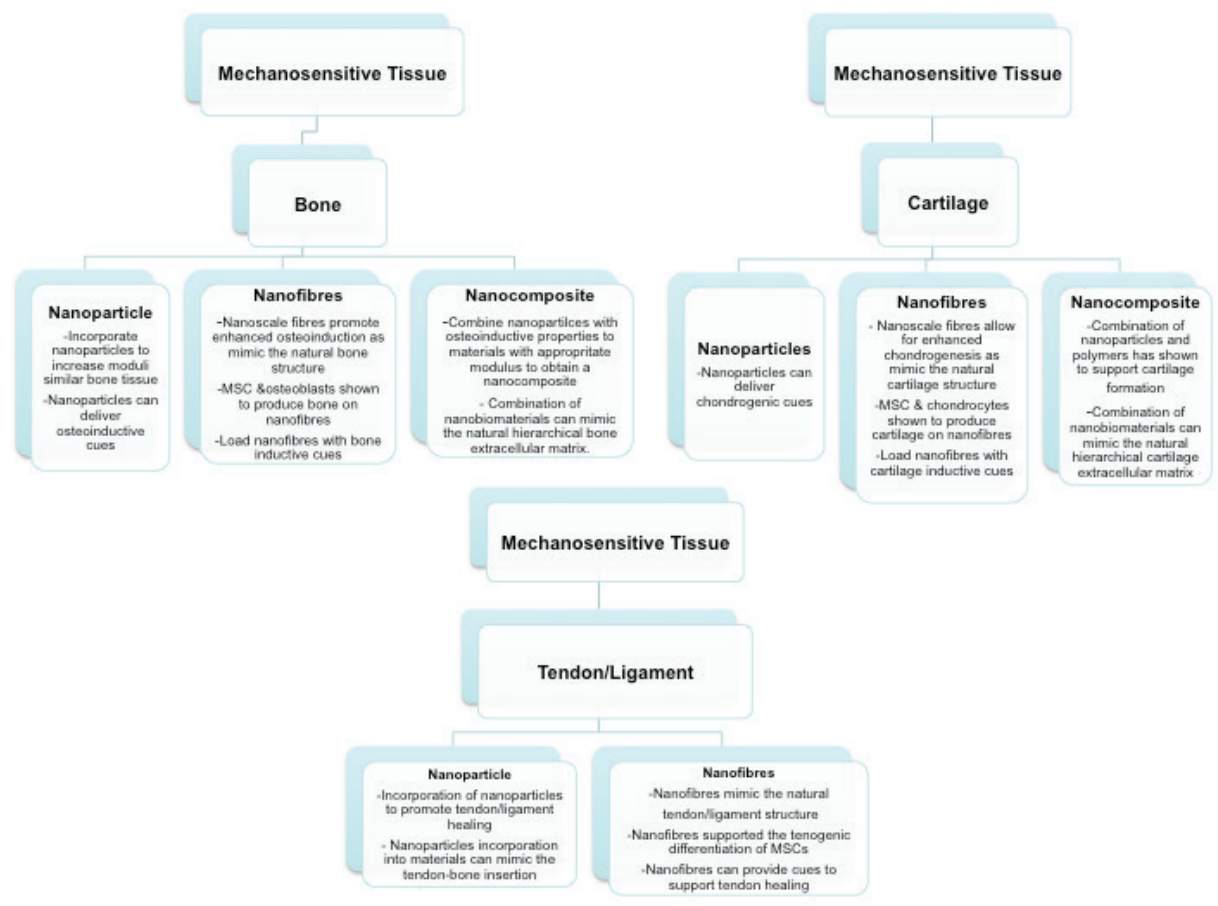

Figure 1. Diagram to illustrate the different nanomaterials used in the regeneration of mechano-sensitive tissues.

\subsection{BONE TISSUE REGENERATION}

Bone tissue is a hierarchical structure consisting of both organic and inorganic components [14]. The organic collagen type I fibres and inorganic HA crystals create a mineralized collagen based-ECM [14]. The complex structure creates a cellular organization, which has an optimal mechanical stiffness and architecture for molecular cues to drive gene expression and protein production [14].

\subsubsection{Clinical need}

There are a number of bone graft substitutes used surgically to repair osseous defects including synthetic, autografts and allografts $[15,16]$. Autografts are the gold standard for the surgical repair of bone defects due to their low immunogenicity and contain all the properties to support good bone regeneration effect $[15,16]$. Autografts contain the ability to induce bone formation including growth factors, osteoprogenitor cells and threedimensional porous matrix $[15,16]$. However, traditionally auto-graft bone grafts are taken from the iliac crest, which causes donor site morbidity, risk of 
scarring, deformity and has several surgical risks including bleeding and infection $[15,16]$. Furthermore, large defects often require more than is available $[15,16]$. Therefore, to overcome such complications synthetic alternative are being investigated and this has initiated the field of bone tissue-engineering.

\subsubsection{Evolution of nanomaterials for bone tissue engineering}

Various nanomaterials have been developed for bone regeneration over the decade including metals, ceramics and polymeric materials $[17,18]$. An ideal bone scaffold material must have good mechanical properties, demonstrate adequate biocompatibility, show low patient morbidity, be easily available to surgeons, cost effective and support bone regeneration [19]. The size of pore and porosity are also important for bone tissue formation [20]. An ideal nanomaterial is aimed to mimic the natural hierarchical structure of bone, while maintaining the mechanical properties [20].

\subsubsection{Nanoparticles (NPs)}

The first type of nanomaterials that have been researched within bone regeneration are NPs [21]. NPs exist in the nanosize range, usually less than $<100 \mathrm{~nm}$ and due to their size can be used to deliver drugs, growth factors and genetic cues [21]. Nanoparticles have been commonly used in regenerative medicine and for bone tissue engineering [22]. The applications of NPs in bone spreads from drug delivery for cancer therapy to treatment of systemic bone diseases, cell labelling and controlling cells responses for bone differentiation $[21,22]$. Ideal properties which need to be incorporated in the design of NPs are to be nontoxic, bio-inert and they should invoke minimal side effects after implantation [23].

The main application of NPs for bone tissue engineering is mimicking the natural hierarchical structure of bone [21,22]. If tissue engineered scaffolds can mimic the native extracellular matrix, cell will be able to adhere, move and support tissue formation. NPs are promising candidates for bone engineering as they can mimic the same size as the HA crystals of the bone ECM and hence can provide these cellular cues [21,22]. Nanoparticle modified composite scaffolds offer far more promises to facilitate bone formation [21,22].

Site specific tissue formation has been a dream of every biomaterial scientist. To realize this ambition various bone inducing growth factors, proteins and drugs has been incorporated with NPs. To enhance the osteogenic differentiation of mesenchymal stem cells (MSCs) several growth factors have been combined with NPs [24]. The enhancement of osteogenic differentiation has shown to be supported by bone morphogenetic proteins (BMPs) in several studies to date [25-27]. Bone-morphogenetic protein-2 (BMP-2) incorporated on NPs has shown to support in vitro osteogenic differentiation and promote bone formation in vivo [28]. BMP-2 and bone-morphogenetic protein-7 
(BMP-7) loaded NPs incorporated onto three-dimensional (3D)-fiber meshes, showed a synergistic effect as a bone forming scaffold [28].

Synthetic stimulators of bone formation have also been incorporated with NPs to promote bone formation which include NPs containing genetic cues [29-31]. Bioglass is a glass that contains biological and physiological functions suitable for the regeneration of osteochondral restoration. A bioactive glass nanoparticle (nBG) poly(lactic acid) (PCL) coating on HA/ $\beta$-tricalcium phosphate (HA/bTCP) scaffold demonstrated upregulation of osteogenic gene expression (Runx-2, collagen I, osteopontin and bone sialoprotein) and alkaline phosphatase activity in adipose derived stem cells (ADSCs) when used in combination with BMP-2 treatment [29]. As the delivery of proteins and growth factors poses challenges in terms of optimization of the release of the bioactive factors, NPs have been researched to act as gene carriers. The transfection approach is a promising strategy for regenerative medicine as it allows long-term expression, causing a potential for a longer therapeutic effect. Several NPs have been used to carry plasmids encoding transcription factors, growth factors and hormones. For example, a BMP-2 encoding plasmid was incorporated into calcium phosphate NPs on alginate hydrogels [30]. As NPs have can have controlled degradability, safety and ability to target the tissue of interest, gene therapy may show a promising future for bone regeneration [31].

Emerging area where NPs are investigated widely is stem cell therapy. It is hoped that stem cells therapy can promote tissue regeneration due to their self-renewal capacity. However, differentiation of stem cells in specific cell type in vivo still remains a challenge. Stem cell tracking by using various NPs such as quantum dots, fluorescence silica NPs and superparamagnetic iron oxide (SPIO) NPs is being used as a tool to evaluate stem cell migration and their phenotypes $[32,33]$. However, there are challenges to be faced once these techniques are optimized for bone tissue engineering. It is important that nanoparticle does not affect the MSCs potential for bone differentiation. Despite gold NPs and SPIO showing the ability to track MSC in vitro and in vivo, some studies have found the NPs to alter osteogenic differentiation, which warrants further investigation [34].

\subsubsection{Nanocomposites for bone tissue engineering}

Native bone consists of nanocomposite structure, ceramic HA particles and a collagen fiber matrix [35]. Hence, nanocomposite scaffolds made of biocompatible polymer and bioactive inorganic NPs have been extensively researched for bone tissue regeneration due to their ability to mimic the natural bone structure, whilst providing excellent mechanical properties and osteoinductive and osteoconductive properties [35-37]. Since natural bone matrix is a composite of biological ceramics (HA) and polymer (collagen) several synthetic and natural biomaterials hybrids have been developed to 
mimic the natural bone structure and provide suitable scaffolds for bone tissue engineering [35-37]. Several bone graft materials have been researched to serve as a carrier to deliver the cells to the injured bone acting as a support to regenerate bone tissue including bioceramic materials, polymers, natural and metallic materials. Bioceramics, including calcium phosphate, bTCP, HA and calcium sulphate have excellent osteoconductive properties [35]. However, their brittle nature often causes such materials to fail as a bone substitutes $[35,36]$. To overcome this problem polymers have been combined with a ceramic material to obtain suitable scaffolds [35,37]. Combining bioactive ceramic particles to polymer materials improves the mechanical properties of the polymeric scaffolds and enhances their bone inductive behaviour [38,39]. Calcium phosphate NPs within PLGA microspheres showed proliferation of osteoblast cells, having a mechanical stiffness that was similar to bone tissue [40].

As bone contains HA particles, many studies have tried to incorporate nano-HA into the polymer matrix to improve the scaffold biological properties and support bone formation in vivo. For example, PCL and nano-HA have shown to improve the mechanical strength of PCL alone and stimulate osteoblast proliferation [41]. After the incorporation of n-HA into poly(lactic-co-glycolic acid) (PLGA) salt leached scaffolds, in vivo mineralisation was formed and osteogenesis was found in the radius defect of rabbits after nine weeks of implantation [42].

Natural polymers have also shown promise as bone graft scaffolds including collagen, silk, hyaluronic acid, gelatin, chitosan (CS) and alginate. Combining bioceramic and natural polymer to create nanocomposites has also showed to support osteogenesis. For example HA, bTCP and calcium phosphate have been incorporated into natural polymers to improve their mechanical properties and osteogenesis capacity [43-45]. Gelatin with HA nanocomposite materials demonstrated good cell adhesion and proliferation of human MSCs as well as good compressive strength [46]. CS has also showed promise as a bone graft substitute due its ability to support cell ingrowth and antibacterial properties showing bone formation when combined with HA [47].

\subsubsection{Nanofibres in bone tissue engineering}

The goal of the scaffold is to provide a 3D environment for cells to attach and form tissue [9]. One particular type of scaffold manufacture that has obtained a great research interest is nanofibres [9]. The development of nanofibres is expanding due to their potential ability to mimic the architecture of extracelluar matrix (ECM) of the native tissues [9]. The major protein of the ECM is collagen which is arranged in nanofibres ranging from $50-500 \mathrm{~nm}$ in diameters which controls cell behaviour and in turn bone formation [9]. In bone, the ECM consists of the mineralised collagen type I fibres, which consists of $90 \%$ collagen [9]. The mineralised collagen fibrils align and arrange in ways 
to form a hierarchical structure and form full bone [9]. Therefore, developing nanofibrous scaffolds to mimic the natural ECM will allow for cell adhesion, proliferation and differentiation and in turn bone formation [9]. Extensive research is being carried out to understand the potential of nanofibrous scaffold for bone tissue engineering [9]. Nanofibres permit ingrowth, proliferation and differentiation of human MSCs downwards osteoblast like cells [9]. Nanofibres ability to promote cell adhesion, proliferation and cell migration is due to their high surface area to volume ratio [9].

There are several methods to generate nanofibres including electrospining, phase separation and self-assembly [9]. However, electrospining has dominated the literature showing to be the most effective way to create nanofibres and stimulate bone formation. Electrospining allows the ability to control the thickness and composition of the nanofibres, porosity of the scaffold simply and effectively, which in turn enables the ability to control bone regeneration $[48,49]$. This technique creates polymer nanofibres by applying a high voltage to a syringe filled with polymer solution. The polymer solution is held at the end of the syringe tube due to surface tension and the electrical potential provides charge to the polymer solution $[48,49]$. The charge repulsion of the polymer solution induces a force that is opposite to the surface tension of the polymer solution $[48,49]$. As the electrical potential increases the surface of the polymer solution elongates to form a conical tube known as the Taylor Cone [48,49]. The critical electrical potential to overcome the surface tension causes the formation of a jet to be ejected from the Taylor Cone and eventually forms orientated nanofibres that are collected on a stationary or rotatory metallic collector. Several materials have found to be suitable for electrospinning including natural and synthetic polymer for bone tissue engineering [48,49].

Various natural polymers and proteins have been electrospun to biomimic ECM matrix for bone regeneration. Collagen has become a very well researched protein and has shown the ability to support several cell types including osteoblasts, chondrocytes, tenocytes and MSCs [50]. CS is another natural biomaterial, which has shown to be a suitable scaffold for musculoskeletal regeneration [51]. CS / poly(ethylene oxide) (PEO) nanofibres demonstrated the ability to support the attachment of osteoblasts and chondrocytes whilst sustaining adequate fibre mechanical and morphology [51]. Gelatin has shown to support the attachment of cells and mimic the ECM, proving to be a candidate for tissue engineering scaffolds. Gelatin has been electrospun with PCL polymer fibrous scaffolds and shown to improve mechanical strength and surface wettability of PCL or gelatin fibres [52]. Fibroin is another well-researched natural biomaterial for nanofibrous scaffolds due its biocompatibility [50]. Fibroin has shown to have adequate fibres length and surface area proving to be suitable for tissue engineering purposes [53].

Electrospun silk fibroin scaffolds which showed high surface area, porosity and interconnectivity, promoted adhesion and proliferation of cells for bone tissue 
engineering applications [54]. Kimetal. investigated the hybrid electrospinning of silk fibroin (SF)/PCL nano/microfibrous composite scaffolds with various compositions [54]. The increments of SF nanofibre content in the composite scaffolds led to a decrease in pore size but did not have an effect of human mesenchymal stem cell (hMSC) differentiation [54]. However, composite scaffolds were better than PCL nanofibres alone and stimulated new bone formation in a rabbit calvarial defect model (Figure 2) [54].
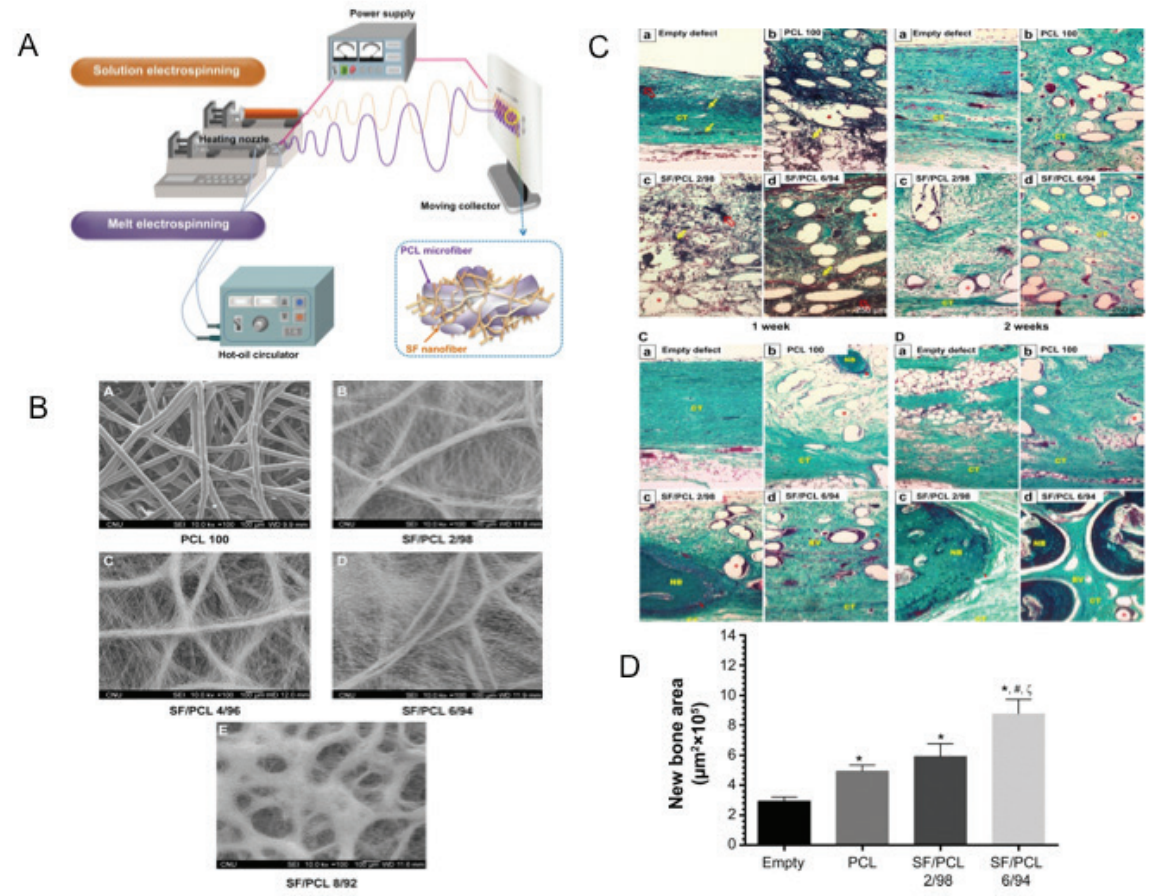

Figure 2. A Diagram of the hybrid electrospinning system and an SF / PCL nano / microfibrous composite scaffold. B Changes in morphology of the PCL microfibrous scaffold and SF / PCL nano / microfibrous composite scaffolds consisting of varying SF-nanofiber content. C Masson's trichrome-stained histological section after implantation. D Quantitative analysis of new bone area at 8 weeks. Taken with permission from [54].

However, electrospun silk scaffolds are limited due to their low mechanical strength [55]. One strategy to overcome this problem was to incorporate HA NPs (HAp) within the nanofibrous scaffolds produced by electrospining [55]. Kim et al. illustrated that it is possible to enhance the mechanical properties of the nanofibres by uniformly dispersing HAp within SF nanofibres [55]. The nanofibres showed peak strengths at the HAp content of 20 wt \% [56]. The 
success of incorporating HA with nanofibres was illustrated by Lai etal. demonstrating that the incorporation of nano HA (nHAp) within a CS / SF scaffold creates favourable microenvironment for bone tissue formation [56]. The osteogenic differentiation of hMSCs was observed during in vivo experiments. The authors demonstrated that histological and immunohistochemical analysis of the retrieved hMSCs/nanofibrous membrane scaffold (NMS) construct 1 and 2 months post implantation indicating that NMS had the potential for bone regeneration and suggested the scaffolds were promising scaffolds for bone tissue engineering [56].

Natural polymers have been found to be difficult to electrospun due to their high viscosity and low solubility [55]. To overcome this, synthetic polymers have been added to the natural polymers to create more compatible electrospun scaffolds including collagen and silk for tissue engineering purposes [57]. For example, PLGA nanofibres with gelatin and nHAP have shown to form biometric scaffolds suitable for bone formation [57]. In addition to natural fibrous scaffolds several synthetic polymers have been electronspun to create suitable scaffolds. A simple and compatible synthetic material PCL has been studied extensively for bone tissue engineering and regenerative medicine as a nanofibrous scaffold. The polymer PCL has been combined with several materials to create biomimetic nanofibres. Biomimetic 3D thick nanofibrous PCL scaffolds demonstrated bone formation in vivo showing the ability to mimic the fibrillar organization of the bone ECM [5]. The combination of PCL/aloevera/silk fibroin-HA nanofibrous scaffolds showed enhanced osteogenic differentiation and mineralisation in comparison to PCL nanofibres alone using adipose stem cells [7]. In addition, PCL nanofibres with phlorotannin, the main component of the brown alga Ecklonia cava has also showed to simulate bone formation of osteoblast like cells (MH63) [58]. Lou et al. demonstrated that nanofibrous poly(L-lactic acid) (PLLA) fibres with diameter of 70-300 nm with $\beta$-TCP improved the mechanical properties and the bioactivity of the PLLA [59]. The modified fibres supported MG-63 osteoblast proliferation, penetration and ECM deposition [59]. Titanium dioxide $\left(\mathrm{TiO}_{2}\right)$ nanofibrous scaffolds demonstrated better cell adhesion and proliferation compared to the flat control surfaces using cell cycle analysis [60]. Expression of stemness markers genes including Nanog3, Rex-1, SOX-2 and nestin of the ADSCs cultured on the control and $\mathrm{TiO}_{2}$ nanofibrous surfaces was also higher on the fibrous surfaces than controls surfaces [60].

To support tissue formation, it has been extensively popular to load nanofibres with bioactive factors to modify the cell environment and mimic the natural ECM niche. Biofactors can be loaded onto nanofibres using several techniques including blending, coaxial spinning and immobilization [60-63]. Blending of the desired factors during electrospining can lead to aggregation homogenous dispersion along the fibres [60-63]. Proteins, molecules and nucleic acid have shown to be blended due nanofibres using these methods. Coaxial electrospining causes the factor to localize to the center of the fibre. This is 
where two solutions are pumped though needles or cylinders and then electrospun to create a fibre [60-63]. Two solutions have different solubility, which prevents the mixing of the fibres during the electrospining process. Lastly, the factor of interest can be adhered or immobilised onto the nanofibre surface using techniques such as chemical functionalization or self assembly [60-63].

Growing evidence of attaching growth factors to nanofibres in vivo to support bone formation has been reported. BMP-2 release from poly(ethylene glycol) (PEG) / PCL showed calvarial bone regeneration after 24 days in a rabbit model [64]. Further support illustrated that BMP-2 could regenerate bone formation on PLGA/HAp nanofibres in the bone defects of tibia in nude mice [65]. Eap et al. illustrated that PCL nanofibrous implant (from $700 \mu \mathrm{m}-1 \mathrm{~cm}$ thick) was functionalized with CS and BMP-7 growth factor using layer-by-layer technology, producing fish scale-like CS/BMP-7 nanoreservoirs [5]. The scaffolds were assessed using in vivo implantation, showing significantly more newly mineralized ECM in the functionalized implant compared to a bare scaffold after 30 days implantation, as shown by histological scanning electron microscopy / energy dispersive X-ray microscopy study and calcein injection [5]. Li et al. demonstrated that nanoparticle-embedded electrospun nanofibre scaffold controlled the dual delivery of BMP-2 and dexamethansone (DEX) and showed that in vivo bone formation (Figure 3) [66]. However, it has become a challenge to finely tune the release of the growth factors to match the physical needs of the in vivo environment to form bone tissue [66]. 
A
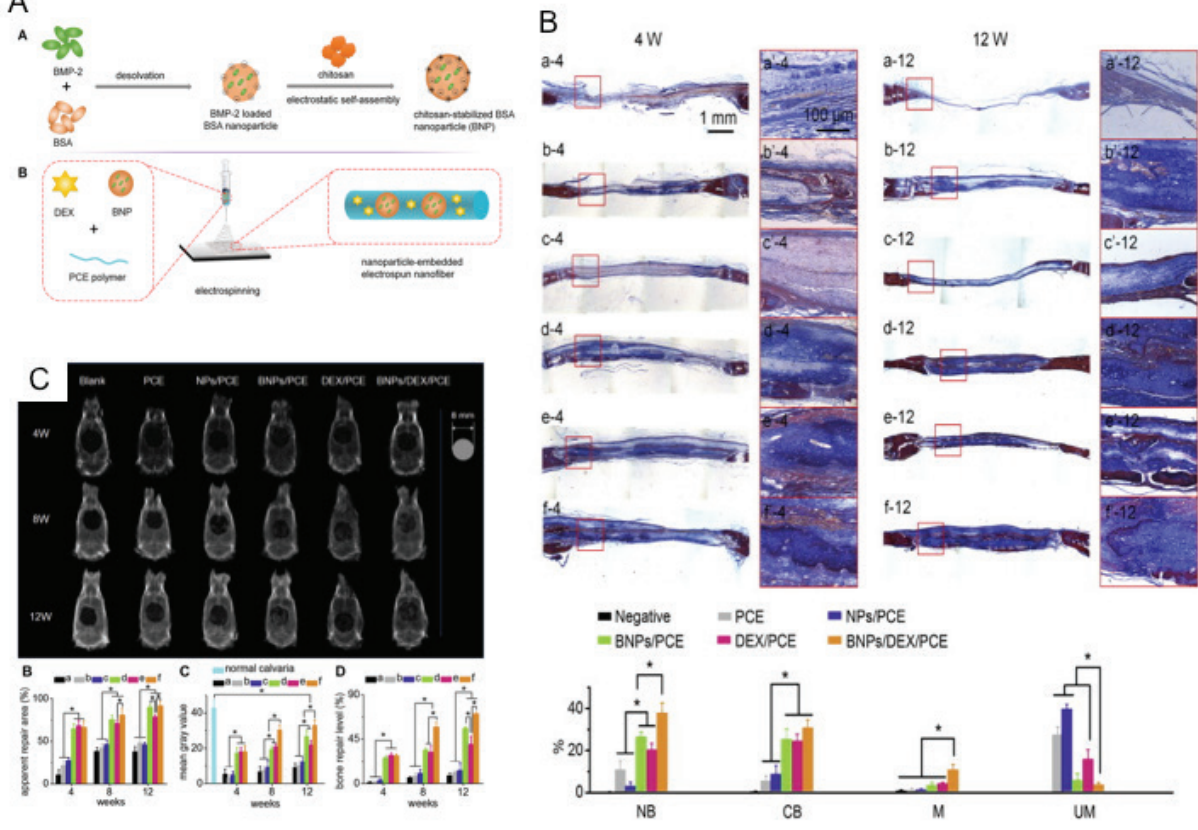

Figure 3. A. Schematic illustration of the fabrication of BMP-2-loaded BSA NPs stabilized with CS (A) and electrospinning of nanoparticle-embedded PCE copolymer nanofibers (B). B. Masson trichrome staining after implanted in situ for 4 and 12 weeks respectively for Control group without material (a), PCE (b), NPs / PCE (c), BNPs / PCE (d), DEX / PCE (e), BNPs / DEX / PCE (f), the red panels are the magnified regions in calvarial panorama. The statistical evaluations calculated from the histological staining pictures of 12 weeks $(n=3)$, in which negative is refer to the blank implanted group, NB represents the newly formed bone area, CB represents the calcified bone area, $\mathrm{M}$ stands for the bone marrow area and UM stands for the undegraded material area. Asterisk mark represents the significant difference between each other, $n=3$ for each group, $p<0.05)$. C. Radiograms of the X-ray detection after implanted in vivo for 4,8 , 12 weeks respectively (A), and the rightmost disk is a calvarial defect template with a diameter of $8 \mathrm{~mm}$. The statistical evaluations of apparent repair area (B), mean gray value (C) and bone repair level (D) were calculated from the radiograms $(n=3)$ for Control group without material (a), PCE (b), NPs / PCE (c), BNPs / PCE (d), DEX / PCE (e), BNPs / DEX / PCE (f). The normal calvaria mean gray value was calculated from an undamaged calvarial bone. Asterisk mark represents the significant difference between each other, $n=3$ for each group, $p<0.05$. Taken with permission from [66].

Biomaterials derived from the decellularisation of mature tissue can also contain the topographical and structural features and influence cellular activity [67]. Gibson etal. maximized this effect and incorporated the present decellularized extracellular matrix (DECM) particles in combination with synthetic nanofibers and examined the ability of these materials to influence 
stem cell differentiation [67]. Nanofibre scaffolds containing up to $10 \%$ particles showed differentiation compared to controls scaffolds after 3 weeks [67].

Extensive research has been carried out into the incorporation of HAp within nanofibres for bone tissue engineering [68]. Poly(L-lactic acid)-co-poly( $\varepsilon$ -caprolactone)/silk fibroin/ascorbic acid/tetracycline hydrochloride (PLACL/SF/AA/TC) and nano-hydroxyapatite (nHA) demonstrated osteogenic differentiation of MSCs, demonstrated using alkaline phosphatase activity, mineralization and double immunofluorescence staining of both CD90 and osteocalcin [68]. Collagen nanofibres with nHA agglomerated by electrospraying improved the adhesion and metabolic activity of MC3T3-E1 osteoblasts [69].

Nanofibres can also act as drug-delivery system for musculoskeletal engineering [77]. Infection of implantable materials remains a long time surgical challenge, with extensive innovation been used to modify materials to prevent bacterial colonisation. It is now possible to include antimicrobial drugs into electrosupn nanofibres. Rapid release of rifampicin from PCL/nanofibres meshes illustrated good antimicrobial resistance to Staphlycoccus epidermidis and prevented biofilm formation. The RF-incorporation nanofibre meshes did not sacrifice the osteogenic properties of the scaffolds showing up regulation of gene expression for preosteoblastic collagen type I and alkaline phosphatase (ALP) [70]. Strontium phosphate incorporation into nanofibres has also shown a potential material for bone tissue engineering. Su et al. utilised PCL nanofibres coated or blended with strontium phosphate and found it supported the osteogenic differentiation of human exfoliated deciduous teeth [71]. Alendronate (ALN) loaded PCL nanofibres scaffolds demonstrated ALP activity, calcium content and osteogenic differentiation of ADSCs genes in vitro. Furthermore, the ALN / PCL scaffolds had a positive effect on bone formation at 8 week after implantation [72]. Further understanding of bioceramics is important for developing smart bone tissue engineering strategies [71].

Carbon nanotubes (CNTs) have excellent mechanical properties, high surface area and light-weight making them ideal scaffold material to be investigated for tissue engineering purposes [73]. CNTs have been incorporated into several polymer materials including PCL, PLA and PLGA to form biomimetic scaffold for bone tissue engineering [74-76]. CNTs have also demonstrated the ability to support osteogenesis when incorporated with several scaffolds materials. Duan et al. illustrated that CNTs incorporated into PLA scaffolds by thermal induced phase separation promoted the osteogenic differentiation of bone marrow mesenchymal stem cells (BMMSCs) [73]. The CNTs enhanced the expression of osteogenesis related proteins as well as the formation of type I collagen [73]. However, the toxicity of CNTs, is still unclear and requires further exploration into the long-term effect on bone remodelling. 


\subsection{CARTILAGE TISSUE REGENERATION}

Cartilage is an avascular tissue that lacks the capacity for repair [77]. Cartilage tissue contains chondrocytes entrapped in an ECM rich in proteoglycan macromolecules and collagen fibrils. Similarly to bone tissue, the hierarchical network structure of cartilage tissue is responsible for its mechanical properties [78]. Tissue engineering approaches have been mainly focused on seeding chondrocytes or MSCs on biomimetic scaffolds for cartilage tissue formation $[77,78]$. Designing suitable scaffolds for cartilage tissue engineering using nanomaterials requires the ability to mimic the native cartilage tissue architecture $[77,78]$. The scaffolds should have similar mechanical properties, biochemical composition as well their physical structure to native cartilage $[77,78]$.

\subsubsection{Clinical need}

Creating biomaterials for cartilage engineering is driven from the thousands of joint procedures including knees and hips that are undertaken each year. Articular cartilage has a very poor ability to repair itself due the poor vascular supply [79]. Currently surgical techniques to restore failure of articular cartilage rely on the drilling or abrasion of the joint to simulate healing or by the delivery of growth factors at the injury site [79]. For larger defects, artificial hip and knee joints are created but these are not ideal for the young patients due to the limited lifespan of the joint [79]. Autografts are available to repair defects but are limited by donor site morbidity, complications from the surgical harvesting site and limited availability of tissue for large defects [79]. These imitations can be overcome by regenerating articular tissue by using tissue-engineering approaches [79].

\subsubsection{Nanoparticles for cartilage tissue engineering}

In a similar strategy to utilize NPs for bone formation, NPs have been incorporated into scaffolds to optimize cartilage formation. NPs can be combined into scaffold materials to provide nanoscale dimensions that mimic the natural ECM of cartilage, providing cellular cues and the ability to support cartilage formation. Furthermore, NPs can deliver growth factors, genetic material and drugs, which can support cartilage formation [81-82].

The incorporation of NPs to support cartilaginous differentiation of stem cells has been documented. For example, PLGA NPs were used to deliver SOX-9 to MSC and demonstrate cartilage formation [80]. Incorporation of aggrecan and cartilage oligomeric matrix protein (COMP) supported chondrogenic differentiation of hMSCs into chondrocytes [81]. Lu etal. similarly demonstrated that NPs could deliver genes to promote stem cells differentiation [82]. A CS scaffold combined with the design of a novel gene-activated matrix (GAM) embedded with hybrid hyaluronic acid (HYA) / CS / plasmid-DNA NPs encoding transforming growth factor 
(TGF)- $\beta 1$ demonstrated an optimal environment for chondrocyte proliferation using histological and biochemical assays [82]. The double release of insulin growth factor-1 (IGF-1) and transforming growth factor-beta 1 (TGF- $\beta 1$ ) from NPs from PLGA scaffolds supported the cartilage formation of MSCs with the collagen type II and aggrecan expression higher than TGF-free scaffolds [83]. Shi et al. demonstrated that adipose derived stem cells transfected with bone morphogentic protein-4 (BMP-4) showed expression of chondrogenesis-related genes and protein and promoted in vivo cartilage formation [84].

\subsubsection{Nanocomposites for cartilage tissue engineering}

The structural and mechanical properties of cartilage are due to the composition and architecture of the ECM. As highlighted earlier, the ECM consists of collagen fibrils and proteoglycan macromoleuclues and non-collagenous proteins to create a hierarchical network [85-86]. Therefore, in a similar approach to designing suitable bone scaffolds, hybrid combinations of biocompatible polymers and ceramic NPs have been investigated for cartilage tissue engineering to mimic the complex architecture of cartilage [85-86].

Numerous scaffold materials have been considered for cartilage repair to date. Both natural and synthetic polymers have been optimized for cartilage engineering. Natural nanoscaffolds have also illustrated to provide a compatible environment of cartilage formation due to the ability to interact with cells and provide optimal cues [85]. The combination of natural polymers and bioceramics has been investigated for cartilage formation. HA with polymer materials has supported cartilage formation [86]. Collagen type II and HA showed a complex three dimensional structure that had good physiochemical and biocompatibility showing histological and immunohistochemical staining for chondrocytes growth and essential protein matrix production [86].

Polymer-HAp composite have also been explored for cartilage repair, including nanofibrous scaffolds. For example, PLGA, poly(vinyl alcohol) (PVA) and PLLA have been electrospun with nHA and found to provide molecular signalling mechanisms ideal for cartilage formation [87-89]. nHA/collagen scaffolds demonstrated the ability to sustain chondrocyte cell attachment, abundant glycosaminoglycan (GAG) synthesis and natural morphology [90]. Collagen / alginate and HA scaffolds have also demonstrated to be promising candidates for osteochondral tissue engineering [91].

Metal and bioceramics materials hybrid combinations have also begun to be investigated for cartilaginous repair [92]. Certain metallic materials have shown to have osteoinductive characteristics and good biocompatibility and hence have been investigated for cartilage engineering. Nanostructured HA with zirconia has shown to repair the articular cartilage defects in rabbits [92]. Titanium has been used in surgical practice for a long-term to replace 
cartilaginous defects. Work by Savaiano et al. showed that PLGA mixed with titanium NPs demonstrated chondrocyte adhesion was higher after the addition of the titanium NPs [93]. Gold NPs have also been incorporated into polymers and shown to improve cartilage formation [94].

Human adipose stem cells (hADSCs) have shown to be useful candidate for tissue engineering purposes due to their angiogenic and wound healing capabilities. Our group has developed polyhedral oligomeric silsesquioxane-poly(carbonate-urea) urethane (POSS-PCU), a novel nanocomposite polymer consisting of PCU with a nanoparticle, POSS incorporated within POSS-PCU supported ADSC survival and proliferation and differentiation into cartilage within the nanoscaffold [95].

\subsubsection{Nanofibres for cartilage tissue engineering}

Electrospinning of scaffolds has gained extensive attention in recent years being able to create suitable scaffolds for cartilage tissue engineering purposes. Nanofibres can facilitate cell behaviour to support cartilage formation due to the ability to create a multi-scale environment, which mimics the ECM of cartilaginous tissue [95-96]. Nanofibrous scaffolds have shown to promote human MSCs attachment, proliferation and also promote chondrogenic differentiation due to the ability to create the complex hierarchical architecture of natural cartilage [95-96].

The size of the nanofibres has shown to effect the attachment of cells and serum proteins [96]. Coburn etal. compared pellet and PVA nanofibres scaffolds cultures and demonstrated enhanced chondrogenic differentiation of mesenchymal stems cells as indicated by increased ECM production and cartilage specific gene expression while also permitting cell proliferation on the fibrous scaffolds [96]. Collagen and PLA nanofibres have also shown to synergistically promote the osteochondral regeneration of MSCs. Osteochondral defects were created in rabbits and repaired by implanting the collagen-nanofiber scaffold. There is an evidence of rapid subchondral bone emergence and cartilage formation using these scaffolds demonstrated by histological, biomechanical testing and microCT testing [97]. He et al. developed a biodegradable hybrid nanofibrous membrane of collagen and poly(L-lactic acid-co- $\varepsilon$-caprolactone) (PLCL, $75: 25$ ) for cartilage engineering. The scaffold demonstrated cartilage like tissue after 12 weeks of implantation in mice [49]. Shafiee et al. illustrated the effects of PLLA / PCL nanofibres on the cartilaginous capacity of nasal septum derived progenitors in vitro [98]. Aligned nanofibres illustrated better chondrogenic differentiation than randomly orientated PLLA / PCL scaffolds as shown by the upregulation of collagen II and aggrecan [98]. In addition, PCL electrospun nanofibres supported the MSC cartilaginous differentiation in a perfusion bioreactor including the cartilage-related genes such as aggrecan, collagen type II, and SOX9 [99]. 
To overcome poor cellular infiltration into nanofibres scaffolds, combination of nano- and microfibrous architecture has been investigated. When used in combination these scaffolds showed cartilage regeneration [100-102]. Poly(D,L-lactide) (PDLLA) nanofibres coated with PDLLA microfibers showed better chondrocyte adhesion than PDLLA films and PDLLA microfibers alone [102].

The incorporation of cartilage specific cues into nanofibres has shown a promising strategy to improving tissue regeneration. Itani et al. investigated PLGA nanofibres with basic fibroblast growth (bFGF) factor-laden particles and an exterior fibrin sealant illustrated optimal cell maintenance and neocartilage formation in mice using nanofibres of 0.8-3.0 $\mu \mathrm{m}$ (Figure 4) [103].
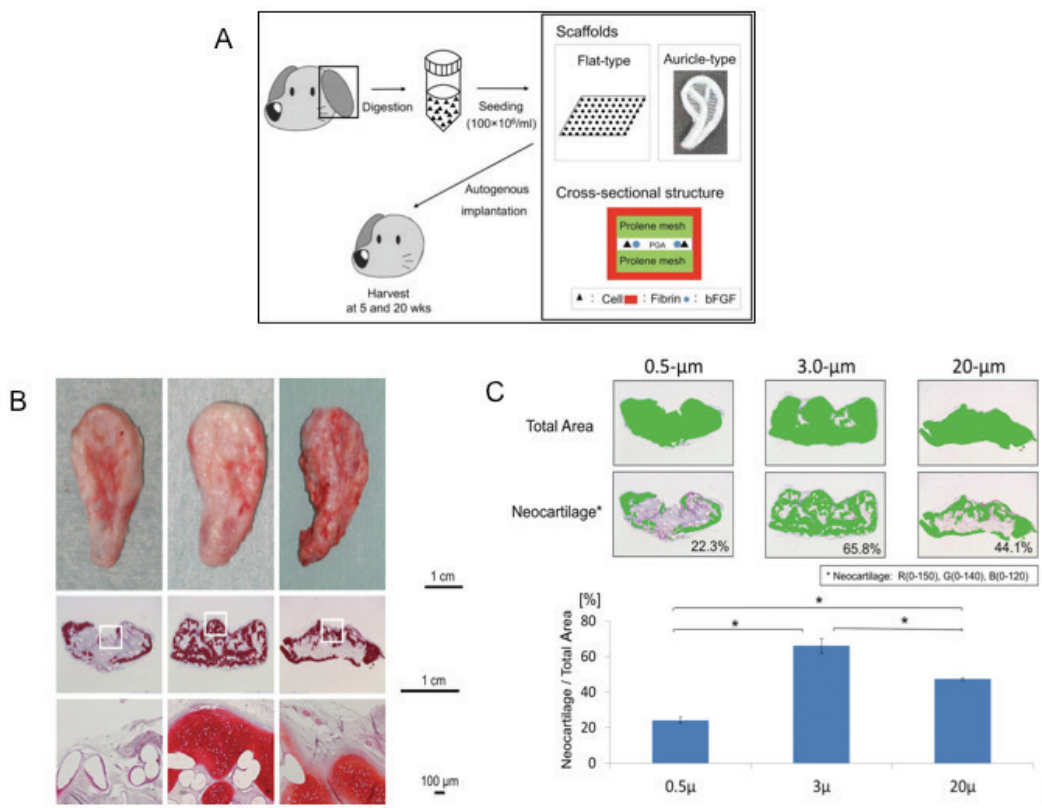

Figure 4. A. Steps taken for the in vivo experiments, from chondrocyte harvest / digestion; to construct creation with incorporation of seeded cells from female beagles, basic fibroblast growth factor (bFGF) particles, and fibrin sealant; to autogenous implantation in a second surgical procedure $20 \mathrm{~h}$ after the chondrocyte

harvest. B. Auricle-type scaffold 20 weeks after implantation, showing gross morphology (top) and safranin 0 staining (center and below) for the (left) 0.5-, (center) 3.0-, and (right) 20- $\mu \mathrm{m}$ groups. C. Quantitation of safranin 0-positive areas in representative cross-sections of samples from the 0.5-, 3.0-, and 20- $\mu \mathrm{m}$ (above and center) groups. The total areas (above) were compared with the positive areas

(neocartilage) identified by image analysis (center) to yield the percentage of neocartilage in the sample. (Below) Bar graph of the percentage of neocartilage at

20 weeks. Bars with error bars are average and standard errors, respectively $\left({ }^{*} \mathrm{p}<0.01 ; \mathrm{n}=6\right.$ in each group; values given as mean $\left.\pm \mathrm{SE}\right)$. Taken with permission from [103]. 
Similarly, PCL nanofibres functionalised with CS and HYA nanofibres cross-linked using carbodiimide chemistry illustrated that the rat bone marrow derived stem cells (rBMSCS) showed differentiation and production of hyaline cartilage proteins including collagen type II and aggrecan [104]. Further support, demonstrated that PCL scaffolds combined with $1 \%$ high-molecular-weight sodium hyaluronate (NHA / MHA), $1 \%$ hyaluronan and $200 \mu \mathrm{g}$ TGF- $\beta 1$; NTGF/MTGF, or $0.1 \%$ bovine serum albumin (BSA) also showed augmentation of MSCs and enabled chondrogenic differentiation [105].

Composite scaffolds have been used to obtain the mechanical properties and the biochemical composition. Zheug etal. demonstrated that natural nanofibrous articular cartilage extracellular matrix (ACECM) can mimic the biochemical composition but be improved when synthetic PLGA is combined to enhance the mechanical strength of ACECM. The composite scaffolds showed better cell proliferation and cell viability than PLGA alone due to the better mechanical properties, making it suitable for cartilage tissue engineering [106]. Nanofibres have also shown to reinforce hydrogels due to their poor mechanical properties and support cartilage formation. The incorporation of electrospun PLCL scaffold with gelatin demonstrated enhanced expression of chondrogenic genes and production of GAGs than those prepared without the hydrogel [107]. Similarly electrospun nanofibres combined with CS / glycerophosphate hydrogels illustrated hyaline cartilage regeneration [107]. Mechanical properties after reinforcement of the hydrogel with silk nanofibres demonstrated significant enhancement of the chondrogenic phenotype, illustrated by elevated GAG content [108]. Fibroin microfibers and collagen hydrogel were demonstrated suitable scaffolds after combined with a pure collagen hydrogel for vascular tissue engineering [109].

CNTs mimic the dimensions of the tissues where cells obtain cues to form cartilage in vivo and hence found to be a good candidate for tissue engineering. CNTs have excellent mechanical properties being suitable for load bearing tissues such as cartilage and bone. Several studies have tried to combine CNTs with nanofibres to create effect scaffolds for musculoskeletal engineering. Chahine et al. illustrated that single walled nanotubes (SWNT) nanocomposite scaffolds could be used in cartilage tissue engineering providing a structural reinforcement of the scaffolds mechanical properties [110]. Chondroyctes tolerated functionalized SWNT with $\mathrm{COOH}$ or -PEG with minimal toxicity of cells and increased collagen II (Col2a1) and fibronectin (Fn) gene expression throughout the culture in nanocomposite constructs, indicative of increased chondrocyte metabolic activity [110]. CNTs incorporated into alginate scaffolds have showed the chondrogenic differentiation of adipose stem cells showing expression of SOX-9, type II collagen [111].

Chondrocytes illustrated enhanced function on electrically highly dispersed CNTs in polycarbonate urethane compared to stimulated pure PCU. Higher amount of Fn was adsorbed on CNT / PCU composite compared PCU alone due 
to increases hydrophilicity and increased nanometer scale roughness [112]. Furthermore, after the incorporation of multi-walled carbon tubes (MWCNTS) into PLLA nanofibres, a drastic mechanical strength and a compression Young's Modulus matching to natural cartilage was observed, which supported cartilage formation [113]. After 2 weeks of culture PLLA scaffolds with MWCNTs the scaffold showed cartilage formation and promising candidate for further exploration for cartilage regeneration [113].

Osteoarthritis is a degenerating disease of the articular joint. Intra-articular injection of pharmalogic agents into the cartilage resistant chondrocytes has left a problem to developed anti-osteoarthritis (anti-OA) medications. Sacchetti et al. described an efficient intra-articular delivery system based on SWCNTs [114]. They showed that PEG-SWCNTS were able to persist in a joint cavity for a suitable amount of time, enter the cartilage matrix and deliver gene inhibitors to the chondrocytes of both healthy and OA mice without causing any systemic side effects [114].

\subsection{TENDON / LIGAMENT REGENERATION}

Tendon and ligament injuries are a common musculoskeletal clinical problem for the young and active patients [114-119]. The economical and social burden that these injuries can cause makes these medical conditions a compelling area of research to date [114-119]. Tendon and ligament have a similar composition and structure in vivo, connecting skeletal muscle to the bone and bone to bone respectively. The ECM, manly composed of collagen type I of both structures has uniaxial aligned structure leading to anisotropic mechanical properties [114-119]. The tendon matrix is composed of tenocytes with continuous matrix remodelling, whilst the ligament matrix composes of fibroblasts / fibrocytes [114-119].

\subsubsection{Clinical need}

Tendon and ligament injuries are caused by trauma, tumour resection and atrophy. Flexor tendon lacerations, of the hand are commonly caused by trauma and pose a significant challenge for the hand surgeon [114-119]. The Achilles tendon, the largest tendon of the body, is often damaged by Achilles tendiosis leading to rupture and a debilitating condition [114-119]. The most severe ligament injury is the Achilles ligament injury, which causes severe impediment to knee function [114-119]. Tendon and ligaments has a low regeneration for self-renewal due its poor vascularity often leading to a scaring mediated healing response and does not restore the biomechanical properties of the native tendon [114-119]. Several surgical techniques are used to restore severe damage. Biological grafts are used to repair damaged tendons but often are unable to provide the optimal mechanical strength [114-119]. Along with the morbidity and functional disability at the donor site tendon, autografts are 
also limited by their availability [114-119]. Allografts are available to improve upon these complications but are limited to tissue rejection and disease transmission. To overcome these problems for tissue replacement synthetic grafts have been researched for tendon engineering [114-119].

Tissue-engineering aims to develop ideal substitutes to restore tendon / ligament function [114-119]. In a similar strategy to bone and cartilage engineering, the ideal scaffold for tendon regeneration must meet the physiological demands of the native tendon but also direct a cellular response to achieve healing [114-119]. Synthetic and biological scaffolds have been investigated for tendon / ligament repair [114-119]. Nanofibres have gained popularity in the field of tendon / ligament due to the ability to biological mimic the in vivo environment. The collagen-rich ECM can be resembled with nanofiber scaffolds due to high surface area-volume ratio, variable fibres and pore size allowing optimal matrix production [118-119].

\subsubsection{Nanofibres for tendon / ligament tissue engineering}

Due to fibrous nature of these tissues, the most common structure, which is used for tissue engineering strategy is fibrous scaffolds. As already highlighted nanofibres ranging from 1-100 mm closely match the size of the ECM fibres making them suitable to produce cellular cues [121-122]. Fibrous scaffolds have gained extensive interest in tendon / ligament regeneration due the high porosity allowing for high cell infiltration rate and the ability to create uniaxial alignment, which mimics the anisotropic structure of the native tissues. Aligned nanofibres have shown to be a suitable scaffold for engineering ligaments and tendon as it mimics the anisotropy of the native tissue [121-122].

Several studies have shown synthetic nanofibres supporting cell adhesion and promising candidates as scaffolds for tendon regeneration [120]. For example, PLGA nanofibres allowed the differentiation of porcine MSCs towards the tenogenic lineage. Silk and PCL nanofibres showed an optimal structure allowing the good fibroblast attachment and a promising scaffold for ligament engineering [121]. Similarly, PCL with random and aligned silk fibres showed large porosity and pore sizes by scanning electron microscopy (SEM), which favoured BMSCs proliferation whilst sustaining satisfactory mechanical properties for tendon repair [122].

Compared to bone marrow stem cells, ADSCs can be harvested using a minimally invasive procedure, are in abundant supply in current populations, have the comparable ability to differentiate into mesenchymal lineages and immunosuppressive capabilities and thus are an attractive stem cell source for the translational point of view for tendon engineering [123]. One study included ADSCs on PLGA, a biodegradable scaffold, with appropriate mechanical properties was tested for its ability to promote tendon healing. The in vitro studies demonstrated that cells remained viable within the scaffold and 
growth factor release was sustained. The in vivo data supported that the cells remained at the injury site 9 days postoperatively and supported tendon healing [123].

Flexor tendon healing remains problematic due to the ability to surrounding cells to create an appropriate healing response. To provide adequate healing, tendon cells at the tendon stumps must migrate, proliferate and then produce ECM. There are many factors to determine optimal tendon behaviour. One factor to consider when designing an optimal fiber for tendon engineering is the orientation of the fibres. Ouyang etal. showed that human tendon progenitor cells (hTSPCs) were spindle shaped and oriented to the direction of the PLLA fibres, however showed tendon specific genes on aligned rather than random fibres [124]. The authors concluded that the orientation of the fibres can affect the hTSPCs differentiation. Lu et al. also showed similar results finding that integrin expression profiles for tenocytes was higher on aligned nanofibres than non-aligned nanofibres [125].

A few biological materials have been utilised as nanofibres to promote tendon regeneration. A recent study, illustrated that collagen and cellulose nanofibres dispersed in a collagen matrix supported human ligament cells and human endothelial cell function [126]. However, the mechanical properties of biological scaffolds have also caused further investigation of hybrid scaffolds [126]. It is also important to highlight the crimped nature of collagen fibrils in tendons and ligaments. The crimped nature of native tendon and ligaments can be mimicked using nanofibres by inducing a crimp when removing the mandrel when rotated at high speed. The resulting crimp structures have shown the same characteristics of the ECM of the tendon and ligament [127].

One strategy that has been implemented is that the ideal scaffold should contain growth factors and thus regulate cellular behaviour and support the formation of the target tissue [128]. Sahoo et al. demonstrated that bFGF releasing nanofibrous scaffolds facilitated BMSC proliferation, upregulated gene expression of tendon/ligament-specific ECM proteins, increased production and deposition of collagen and tenascin- $\mathrm{C}$, reduced multi-potency of the BMSCs and induced tendon / ligament-like fibroblastic differentiation, indicating their potential in tendon / ligament tissue engineering applications [128]. Platelet derived growth factor (PDGF) releasing nanofibres affected the cellular activity of ADSCs. It was observed that the aligned nanofibres significantly enhanced the tenogenic differentiation of ADSCs comparing to the randomly aligned tendon markers such as tenomodulin and scleraxis [129]. Similarly, Liu et al. observed that electrospun PLLA copolymer fibres with bFGF enhanced cell proliferation as well as tendon healing [130]. A very successful example of loading NPs with growth factors for tendon healing was demonstrated using dextran glass NPs. Liu et al. trialled bFGF loaded dextran glass nanoparticle on PLLA fibres and found evidence of tendon healing in vivo (Figure 5) [130]. 
A

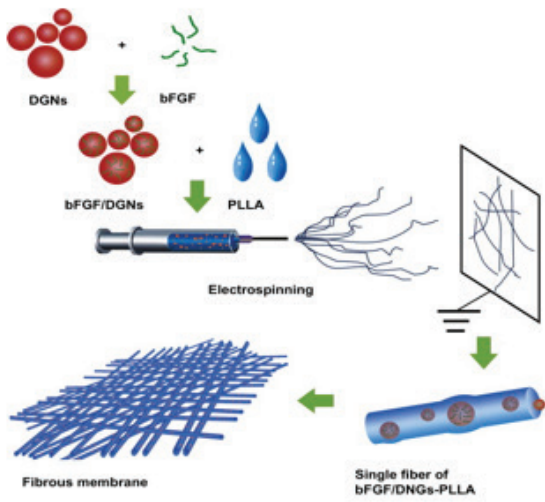

B

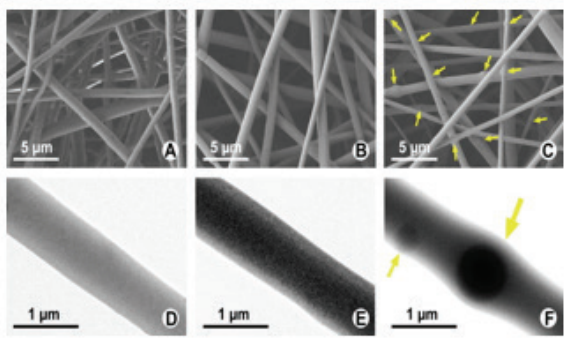

C

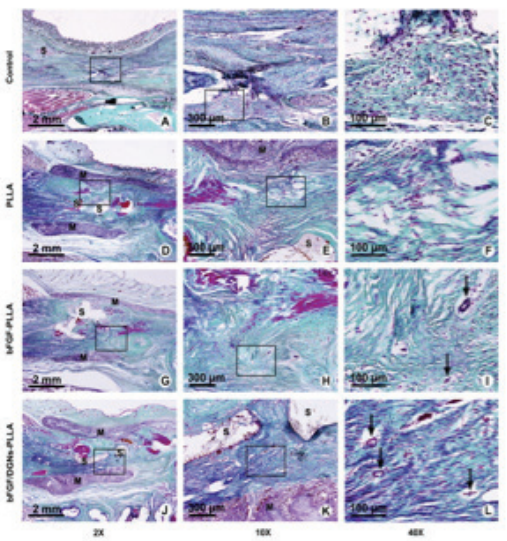

D

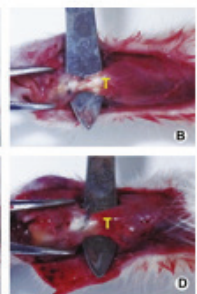

Figure 5. A. After incorporation of bFGF into DGNs by a freeze-induced particleforming process, a bFGF / DGNs-loaded PLLA fibrous membrane was fabricated by electrospinning, producing a membrane which can release bFGF sustainably and has the ability to secure the biological activity of bFGF. B. SEM images of electrospun PLLA (A), bFGF-PLLA (B) and bFGF / DGNs-PLLA (C) fibers. TEM images of electrospun PLLA (D), bFGF-PLLA (E) and bFGF / DGNs-PLLA (F) fibers. Yellow arrows indicate the DGNs embedded into fibers with thickened regions of PLLA visible around them. C. Masson's trichrome staining of an untreated repair site $(A, B, C)$ and a repair site wrapped with the PLLA membrane (D, E, F), bFGF-PLLA membrane (G, H, I) and bFGF / DGNs-PLLA

membrane (J, K, L). Sutured site $(S)$ and materials $(M)$ could be detected. Regional magnification of the repair sites $(A, D, G, J)$ showed the repaired sites of the tendon $(B$,

$E, H, K)$ while regional magnification of the repaired sites $(B, E, H, K)$ revealed the arrangement of the collagen fibers (C, F, I, L). Arrows indicate the capillary vessels, and the square is the area of magnification of the subsequent photo. D. Gross evaluation of a rat model of Achilles tendon surgery after 21 days. (A) Untreated control group; (B)

group treated with the PLLA membrane; (C) group treated with the bFGF-PLLA membrane; (D) group treated with the bFGF / DGNs-PLLA membrane. Tendon (T) was indicated in the pictures. Taken with permission from [130].

The incorporation of NPs to nanofibres has also been investigated for tendon tissue engineering. Infection is surgical complications, which can prevent tendon healing. Silk fibres impregnated with NPs have shown to improve the sterility against multiple microbes. Silk fibres were coated with CS impregnated with silver NPs (Ag-C-SF), which supported anti-microbial 
activity and improved thermal stability, showing potential to be explored as a wound dressing and for tendon reconstruction [131].

Flexor tendon repair is often complicated by the formation of adhesions. Zhou et al. demonstrated a sophisticated in vitro and in vivo transfection experiments demonstrated using NPs / plasmid complex caused less severe adhesions, but did lower the tendon strength of the tendon healing [132]. They used micro RNA (miRNA) plasmids complexed with PLGA NPs to form nanoparticle / TGF- $\beta 1$ miRNA plasmid (nanoparticle / plasmid) complexes [132]. The repair of Achilles tendon healing has also been investigated using mesporous silica NPs in vivo using a rat's Achilles tendon [133].

Another important element of tendon engineering is the ability to engineer complex tissue such as the tendon-bone insertion site that required strong materials with mineralised and unmineralised tissues with varied strength and stiffness. Kolluru et al. demonstrated that PLGA nanofibres which incorporated HA material demonstrated the highly desirable hardening behaviour even in the presence of surface mineralization [134]. The nanofibres provided a $200 \%$ increase in fiber strength and $100 \%$ elongation for thin and strongly bound mineral coatings [134]. An important feature of electrospining is that the scaffolds can have graded physical and chemical properties. For the tendon-bone interface this is vital as this allows the mechanical and structural properties to be graded and mimic the in vivo tissue insertion site. The ability to control the mineral deposition of the scaffold may be another technique to recapitulate the tendon-bone interface. A few studies have aimed to grade the mineralisation along the nanofibres, which has resulted in good cell attachment [135-137].

\subsection{SUMMARY OF DIFFERENT APPLICATIONS}

\subsubsection{Future challenges and prospectives}

Mechano sensitive and load bearing tissues such as bone, cartilage, tendon or ligament are natural nanocomposite with an hieratical structure at nanoscale. Thus, nanomaterials are being thoroughly investigated as scaffolds for tissue engineering these musculoskeletal tissues. For bone tissue engineering, a number of synthetic and natural polymers have been identified as biomaterials for consideration. The use of polymer-ceramic materials remains a very interesting area of research as bone is composed of inorganic HA crystal and organic materials. However, the optimal polymer-ceramic hybrid is unknown and deserves further research interest for bone regeneration. Several polymers have been considered for cartilage, with hybrid materials being the likely candidate to support regeneration. Natural-based and synthetic based blends are being trialed to optimize the mechanical properties and cell interactions required for regeneration. However, despite many composite 
materials being considered, many are far away from being used in clinical trials and require further optimisation.

In particular, electrospun nanofibres have shown the ability to support cell adhesion and differentiation for bone, cartilage and tendon engineering. The similarities to the natural ECM of nanofibres show that the high surface area and porosity is a favorable environment to support tissue formation for regenerative engineering purposes. The exact fiber diameter and orientation to match the ECM is still not clearly understood, as well as the correct combination of micro and nanofibres. As one material type cannot provide all the required characteristics of the ideal scaffold, it is likely hybrid scaffolds will be further explored for musculoskeletal applications. Electrospining is also a promising candidate for regenerative scaffolds due to ability to combine the appropriate growth factor. However, the most appropriate growth factor, peptide or protein to support MSC differentiation for cartilaginous and bone formation is also a need to be further explored.

It is likely in the near future to have multi-component scaffolds, which mimic tendon, cartilage and bone tissue in regards to structure and functionality. Further research, to optimise fabrication of porous scaffolds with tailored properties will be explored to achieve smart materials suitable for musculoskeletal regeneration.

\section{REFERENCES}

1. A.S. Brydone, D. Meek, S. Maclaine. Proc. Inst. Mech. Eng. 224 (2020) 1329-1343.

2. R. James, M. Deng, C. Laurencin, S. Kumbar. Front. Mater. Sci. 5 (2011) 342-357.

3. A.P. Mathew, K. Oksman, D. Pierron, M.F. Harmand. Macromol. Biosci. 13 (2013) 289-298.

4. M. Kim, G. Kim. Carbohydr Polym. 90 (2012) 592-601.

5. S. Eap, D. Morand, F. Clauss, O. Huck, J.F. Stoltz, J.C. Lutz, J.E. Gottenberg, N. Benkirane-Jessel, L. Keller, F. Fioretti. Biomed. Mater. Eng. 25 (1 Suppl) (2015) 79-85

6. P. Bhattacharjee, B. Kundu, D. Naskar, T.K. Maiti, D. Bhattacharya, S.C. Kundu. Biopolymers. 103 (2015) 271-84.

7. S. Shanmugavel, V.J. Reddy, S. Ramakrishna, B. Lakshmi, V.G. Dev. J. Biomater. Appl. 29 (2013) 46-58.

8. A. Ardeshirylajimi, M. Mossahebi-Mohammadi, S. Vakilian, L. Langroudi, E. Seyedjafari, A. Atashi, M. Soleimani. Cell Prolif. 48 (2015) 47-58.

9. L. Ghasemi-Mobarakeh, M.P. Prabhakaran, P. Balasubramanian, G. Jin, A. Valipouri, S. Ramakrishna. J. Nanosci. Nanotechnol. 13 (2013) 4656-4657.

10. T.G. Scott, G. Blackburn, M. Ashley, I.S. Bayer, A. Ghosh, A.S. Biris, A. Biswas. J. Nanosci. Nanotechnol. 13 (2013) 1-22.

11. N.G. Sahoo, Y.Z. Pan, L. Li, C.B. He. Nanomedicine (Lond.) 8 (2013) 639-653. 
12. M.H. Hettiaratchi, T. Miller, J.S. Temenoff, R.E. Guldberg, T.C. McDevitt. Biomaterials 35(25) (2014) 7228-7238.

13. G. Gainza, S. Villullas, J.L. Pedraz, R.M. Hernandez, M. Igartua. Nanomedicine 11(6) (2015) 1551-1573.

14. G.G. Walmsley, A. McArdle, R. Tevlin, A. Momeni, D. Atashroo, M.S. Hu, A.H. Feroze, V.W. Wong, P.H. Lorenz, M.T. Longaker, D.C. Wan. J. Biomed. Nanotechnol. 10 (2014) 959-969.

15. M. Hosseinkhani, D. Mehrabani, M.H. Karimfar, S. Bakhtiyari, A. Manafi, R. Shirazi. World J. Plast. Surg. 3 (2014) 3-7.

16. M. Mravic, B. Péault, A.W. James. Biomed. Res. Int. 2014 (2014) 865270.

17. S.M. Zakaria, S.H. Sharif Zein, M.R. Othman, F. Yang, J.A. Jansen. Tissue Eng. Part B Rev. 19 (2013) 431-441.

18. I. Martin, D. Wendt, M. Heberer. Trends Biotechnol. 22 (2004) 80-86.

19. F.J. O’Brien. Mater. Today 14 (2011) 88-95.

20. A.C. Jones, C.H. Arns, A.P. Sheppard, D.W. Hutmacher, B.K. Milthorpe, M.A. Knackstedt. Biomaterials 28 (2007) 2491-2504.

21. T.J. Webster, E.S. Ahn. Adv. Biochem. Eng. Biotechnol. 103 (2007) 275-308.

22. A. Tautzenberger, A. Kovtun, A. Ignatius. Int. J. Nanomed. 7 (2012) 4545-4557.

23. A. Bhirde, J. Xie, M. Swierczewska, X. Chen. Nanoscale 3 (2011) 142-153

24. S.E. Kim, O. Jeon, J.B. Lee, M.S. Bae, H.J. Chun, S.H. Moon, I.K. Kwon. J. Biomed. Sci. 15 (2008) 771-777.

25. J. Guan, J. Zhang, Z. Zhu, X. Niu, S. Guo, Y. Wang, C. Zhang. Stem Cell. Res. Ther. 6 (2015) 5.

26. H. Lysdahl, A. Baatrup, C.B. Foldager, C. Bünger. Biores. Open Access. 3 (2014) 278-285.

27. Y. Açil, A.A. Ghoniem, J. Wiltfang, M. Gierloff. J. Craniomaxillofac. Surg. 42 (2014) 2002-2009.

28. P. Yilgor, K. Tuzlakoglu, R.L. Reis, N. Hasirci, V. Hasirci. Biomaterials 30 (2009) 3551-3559.

29. Z. Lu, S.I. Roohani-Esfahani, J. Li, H. Zreiqat. Nanomedicine 11 (2015) 219-228.

30. M.D. Krebs, E. Salter, E. Chen, K.A. Sutter, E. Alsberg. J. Biomed. Mater. Res. A 92 (2010) 1131-1138.

31. $\quad$ O.C. Farokhzad. Expert Opin. Drug Deliv. 5 (2008) 927-929.

32. R.J. Byers, E.R. Hitchman. Prog Histochem Cytochem. 45 (2011) 201-237.

33. B.J. Muller-Borer, M.C. Collins, P.R. Gunst, W.E. Cascio, A.P. Kypson. J. Nanobiotechnology 5 (2007) 9.

34. Y.C. Chen, J.K. Hsiao, H.M. Liu, I.Y. Lai, M. Yao, S.C. Hsu, B.S. Ko, Y.C. Chen, C.S. Yang, D.M. Huang. Toxicol. Appl. Pharmacol. 245 (2010) 272-279.

35. T.L. Arinzeh, T. Tran, J. Mcalary, G. Daculsi. Biomaterials 26 (2005) 3631-3638.

36. C. Wang, Y. Xue, K.L. Lin, J.X. Lu, J. Chang. J. Sun. Acta Biomater. 8 (2012) 350-360.

37. D.M. Luo, L. Sang, X.L. Wang, S.M. Xu, X.D. Li. Mater. Lett. 65 (2011) 2395-2397.

38. D.T.J. Barone, J.M. Raquez, P. Dubois. Polym. Adv. Technol. 22 (2011) 463-475.

39. T. Serizawa, T. Tateishi, M. Akashi. J. Biomater. Sci. Polym. Ed. 14 (2003) 653-663.

40. J.R. Popp, K.E. Laflin, B.J. Love, A.S. Goldstein. J. Tissue Eng. Regen. Med. 6 (2012) 12-20. 
41. S.J. Heo, S.E. Kim, J. Wei, Y.T. Hyun, H.S. Yun, D.H. Kim, J.W. Shin, J.W. Shin. J. Biomed. Mater. Res. A 89 (2009) 108-116.

42. D.X. Wang, Y. He, L. Bi, Z.H. Qu, J.W. Zou, Z. Pan, J.J. Fan, L. Chen, X. Dong, X.N. Liu, G.X. Pei, J.D. Ding. Int. J. Nanomedicine 8 (2013) 1855-1865.

43. R.J. Kane, H.E. Weiss-Bilka, M.J. Meagher, Y. Liu, J.A. Gargac, G.L. Niebur, D.R. Wagner, R.K. Roeder. Acta Biomater. (2015) 16-25.

44. Y.J. Hong, J.S. Chun, W.K. Lee. Colloids Surf. B Biointerfaces 83 (2011) 245-253.

45. J. Ramier, D. Grande, T. Bouderlique, O. Stoilova, N. Manolova, I. Rashkov, V. Langlois, P. Albanese, E. Renard. J. Mater. Sci. Mater. Med. 25 (2014) 1563-1575.

46. T.M. Mututuvari, A.L. Harkins, C.D. Tran. J. Biomed. Mater. Res. A 101 (2013) 3266-3277.

47. R.L. Dahlin, F.K. Kasper, A.G. Mikos. Tissue Eng. Part B Rev. 17 (2011) 349-364.

48. M.P. Prabhakaran, L. Ghasemi-Mobarakeh, S. Ramakrishna. J. Nanosci. Nanotechnol. 11 (2011) 3039-3057.

49. X. He, W. Fu, B. Feng, H. Wang, Z. Liu, M. Yin, W. Wang, J. Zheng. Regen Med. 8 (2013) 425-436.

50. N. Bhattarai, D. Edmondson, O. Veiseh, F.A. Matsen, M. Zhang. Biomaterials 26 (2005) 6176-6184.

51. Y. Zhang, H. Ouyang, C.T. Lim, S. Ramakrishna, Z.-M. Huang. J. Biomed. Mater. Res. Part B: Appl. Biomater. 72B (2005) 156-165.

52. H.J. Jin, J. Chen, V. Karageorgiou, G.H. Altman, D.L. Kaplan. Biomaterials 25 (2004) 1039-1047.

53. B.M. Min, G. Lee, S.H. Kim, Y.S. Nam, T.S. Lee, W.H. Park. Biomaterials 25 (2004) 1289-1297.

54. B.S. Kim, K.E. Park, M.H. Kim, H.K. You, J. Lee, W.H. Park. Int. J. Nanomedicine 9 (10) (2015) 485-502.

55. H. Kim, L. Che, Y. Ha, W. Ryu. Mater. Sci. Eng. C Mater. Biol. Appl. 40 (2014) 324-335.

56. G.J. Lai, K.T. Shalumon, J.P. Chen. Int. J. Nanomedicine 10 (2015) 567-584.

57. D. Li,H. Sun, L. Jiang, K. Zhang, W. Liu, Y. Zhu, J. Fangteng, C. Shi, L. Zhao, H. Sun, B. Yang. ACS Appl. Mater. Interfaces. 6 (2014) 9402-9410.

58. W. Ji, Y. Sun, F. Yang, J.J. van den Beucken, M. Fan, Z. Chen, J.A. Jansen. Pharm. Res. 28 (2011) 1259-1272.

59. T. Lou, X. Wang, G. Song, Z. Gu, Z. Yang. Int. J. Biol. Macromol. 69 (2014) 464-470.

60. A.W. Tan, L. Tay, K.H. Chua, R. Ahmad, S.A. Akbar, B. Pingguan-Murphy. Int. J. Nanomedicine 9 (2014) 5389 -5401.

61. N.G. Rim, C.S. Shin, H. Shin. Biomed. Mater. 8 (2013) 014102.

62. D.R. Nisbet, J.S. Forsythe, W. Shen, D.I. Finkelstein, M.K. Horne. J. Biomater. Appl. 24 (2009) 7-29.

63. A. Ferrand, S. Eap, L. Richert, S. Lemoine, D. Kalaskar, S. Demoustier-Champagne, H. Atmani, Y. Mély, F. Fioretti, G. Schlatter, L. Kuhn, G. Ladam, N. Benkirane-Jessel. Macromol. Biosci. 14 (2014) 45-55.

64. H. Zhu, D. Yu, Y. Zhou, C. Wang, M. Gao, H. Jiang, H. Wang. J. Biomed. Mater. Res. B Appl. Biomater. 101 (2013) 541-52.

65. H. Nie, M.L. Ho, C.K. Wang, C.H. Wang, Y.C. Fu. Biomaterials 30 (2009) $892-901$. 
66. L. Li, G. Zhou, Y. Wang, G. Yang, S. Ding, S. Zhou. Biomaterials 37 (2015) 218-229.

67. M. Gibson, V. Beachley, J. Coburn, P.A. Bandinelli, H.Q. Mao, J. Elisseeff. Biomed. Res. Int. 2014 (2014) 469120.

68. C. Gandhimathi, J.R. Venugopal, A.Y. Tham, S. Ramakrishna, S.D. Kumar. Mater. Sci. Eng. C Mater. Biol. Appl. 49 (2015) 776-785.

69. N. Ribeiro, S.R. Sousa, C.A. van Blitterswijk, L. Moroni, F.J. Monteiro. Biofabrication 6 (2014) 035015.

70. X.N. Chen, Y.X. Gu, J.H. Lee, W.Y. Lee, H.J. Wang. Eur. Cell. Mater. 24 (2012) 237-248.

71. W.T. Su, P.S. Wu, T.Y. Huang. Mater.Sci. Eng. C Mater. Biol. Appl. 46 (2015) 427-434.

72. Y.P. Yun, S.J. Kim, Y.M. Lim, K. Park, H.J. Kim, S.I. Jeong, S.E. Kim, H.R. Song. J. Biomed. Nanotechnol. 10 (2014) 1080-1090.

73. S. Duan, X. Yang, F. Mei, Y. Tang, X. Li, Y. Shi, J. Mao, H. Zhang, Q. Cai. J. Biomed. Mater. Res. A 103 (2015) 1424-1435.

74. S.Y. Kim, J.Y. Hwang, J.W. Seo, U.S. Shin. J. Colloid. Interface Sci. 442 (2015) 147-153.

75. J. Alam, M. Alam, M. Raja, Z. Abduljaleel, L.A. Dass. Int. J. Mol. Sci. 15 (2014) 19924-3.

76. J. Xu, Y. Xie, H. Zhang, Z. Ye, W. Zhang. Colloids Surf. B Biointerfaces 123 (2014) 907-15.

77. S.W. O'Driscoll. J. Bone Joint Surg. Am. 80 (1998) 1795-1812.

78. J.S. Temenoff, A.G. Mikos. Biomaterials. 21 (2000) 431-440.

79. M. Falah, G. Nierenberg, M. Soudry, M. Hayden, G. Volpin. nt Orthop. 34 (2010) 621-630.

80. J.H. Kim, J.S. Park, H.N. Yang, D.G. Woo, S.Y. Jeon, H.-J. Do, H.-Y. Lim, J.M. Kim, K.-H. Park. Biomaterials 32 (2011) 268-278.

81. S.Y. Jeon, J.S. Park, H.N. Yang, D.G. Woo, K.H. Park. Stem Cells Dev. 23 (2014) 305-317.

82. H. Lu, L. Lv, Y. Dai, G. Wu, H. Zhao, F. Zhang. PLoS One 8 (7) (2013) e69950.

83. A.B. Ertan, P. Yılgor, B. Bayyurt, A.C. Calıkoğlu, C. Kaspar, F.N. Kök, G.T. Kose, V. Hasirci. J. Tissue Eng. Regen. Med. 7 (2013) 149-160.

84. J. Shi, X. Zhang, J. Zhu, Y. Pi, X. Hu, C. Zhou, Y. Ao. Arthroscopy 29 (2013) 2001-2011.

85. S. Yamane, N. Iwasaki, Y. Kasahara, K. Harada, T. Majima, K. Monde, S. Nishimura, A. Minami. J. Biomed. Mater. Res. A 81 (2007) 586-593.

86. Z. Yang, Z. Chen, K. Liu, Y. Bai, T. Jiang, D. Feng, G. Feng. Experimental study on tissue engineered cartilage complex three-dimensional nano-scaffold with collagen type II and hyaluronic acid in vitro. Zhongguo Xiu Fu Chong Jian Wai Ke Za Zhi 27 (2013) 1240-1245.

87. L. Chen, W.M. Zhu, Z.Q. Fei, J.L. Chen, J.Y. Xiong, J.F. Zhang, L. Duan, J. Huang, Z. Liu, D. Wang, Y. Zeng. BioMed. Res. Int. 2013 (2013) 412745.

88. Y. Guo, J. Guo, D. Bai, H. Wang, X. Zheng, W. Guo, W. Tian. Artif Organs 38 (2014) 580-586.

89. W. Zhu, K. Chen, W. Lu, Q. Sun, L. Peng, W. Fen, H. Li, Y. Ou, H. Liu, D. Wang, Y. Zeng. In Vitro Cell Dev. Biol. Anim. 50 (2014) 214-220.

90. L. Jia, Z. Duan, D. Fan, Y. Mi, J. Hui, L. Chang. Mater. Sci. Eng. C Mater. Biol. Appl. 33 (2013) 727-734. 
91. E. Quinlan, A. López-Noriega, E. Thompson, H.M. Kelly, S.A. Cryan, F.J. O'Brien. J. Control Release 198 (2015) 71-79.

92. A. Sotoudeh, A. Jahanshahi, M.A. Takhtfooladi, A. Bazazan, A. Ganjali, M.P. Harati. Acta Cir. Bras. 28 (2013) 340-345.

93. J.K. Savaiano, T.J. Webster. Biomaterials 25(7-8) (2004) 1205-1213.

94. S.H. Hsu, H.J. Yen, C.L. Tsai. Artif. Organs 31 (2007) 854-868.

95. L. Guasti, B. Vagaska, N.W. Bulstrode, A.M. Seifalian, P. Ferretti. Nanomedicine 10 (2014) 279-289

96. J.M. Coburn, M. Gibson, S. Monagle, Z. Patterson, J.H. Elisseeff. Proc. Natl. Acad. Sci. U.S.A 109 (2012) 10012-7.

97. S. Zhang, L. Chen, Y. Jiang, Y. Cai, G. Xu, T. Tong, W. Zhang, L. Wang, J. Ji, P. Shi, H.W. Ouyang. Acta Biomater. 9 (2013) 7236-7247.

98. A. Shafiee, E. Seyedjafari, E. Sadat Taherzadeh, P. Dinarvand, M. Soleimani. J. Ai. Mater. Sci. Eng. C Mater. Biol. Appl. 40 (2014) 445-454.

99. M.L. Alves da Silva, A. Martins, A.R. Costa-Pinto, P. Costa, S. Faria, M. Gomes, R.L. Reis, N.M. Neves. Biomacromolecules 13(11) (2010) 3228-3236.

100. L. Koepsell, L. Zhang, D. Neufeld, H. Fong, Y. Deng. Macromol. Biosci. 11 (2011) 391-399.

101. E.J. Levorson, P. Raman Sreerekha, K.P. Chennazhi, F.K. Kasper, S.V. Nair, A.G. Mikos. Biomed. Mater. 8 (2013) 014103.

102. I. Wimpenny, N. Ashammakhi, Y. Yang. J. Tissue Eng. Regen. Med. 6 (2012) 536-549.

103. Y. Itani, S. Asamura, M. Matsui, Y. Tabata, N. Isogai. Plast. Reconstr. Surg. 133 (2014) 805e-813e.

104. P. Lee, K. Tran, W. Chang, N.B. Shelke, S.G. Kumbar, X. Yu. J. Biomed. Nanotechnol. 10 (2014) 1469-1479.

105. J.C. Schagemann, S. Paul, M.E. Casper, J. Rohwedel, J. Kramer, C. Kaps, H. Mittelstaedt, M. Fehr, G.G. Reinholz. J. Biomed. Mater. Res. A 101 (2013) 1620-1628.

106. X. Zheng, F. Yang, S. Wang, S. Lu, W. Zhang, S. Liu, J. Huang, A. Wang, B. Yin, N. Ma, L. Zhang, W. Xu, Q. Guo. J. Mater. Sci. Mater. Med. 22 (2011) 693-704.

107. M. Kim, B. Hong, J. Lee, S.E. Kim, S.S. Kang, Y.H. Kim, G. Tae. Biomacromolecules 13 (2012) 2287-2298.

108. F. Mirahmadi, M. Tafazzoli-Shadpour, M.A. Shokrgozar, S. Bonakdar. Mater. Sci. Eng. C Mater. Biol. Appl. 33 (2013) 4786-4794.

109. M.A. de Moraes, E. Paternotte, D. Mantovani, M.M. Beppu. Macromol. Biosci. 12 (2012) 1253-1264.

110. N.O. Chahine, N.M. Collette, C.B. Thomas, D.C. Genetos, G.G. Loots. Tissue Eng. Part A 20 (2014) 2305-2315.

111. A. Valiani, B. Hashemibeni, E. Esfandiary, M.M. Ansar, M. Kazemi, N. Esmaeili. Int. J. Prev. Med. 5 (2014) 825-834.

112. D. Khang, G.E. Park, T.J. Webster. J. Biomed. Mater. Res. A 86 (2008) 253-260.

113. B. Holmes, N.J. Castro, J. Li, M. Keidar, L.G. Zhang. Nanotechnology 24 (2013) 365102. 20.

114. C. Sacchetti, R. Liu-Bryan, A. Magrini, N. Rosato, N. Bottini, M. Bottini. ACS Nano. 8(12) (2014) 2280-2291.

115. S. Font Tellado, E. Rosado Balmayor, M. Van Griensven. Adv. Drug Deliv. Rev. 2015 Mar 14.

116. B.D. Smith, D.A. Grande. Nat. Rev. Rheumatol. 11(4) (2015) 213-222. 
117. A.J. Lomas, C.N. Ryan, A. Sorushanova, N. Shologu, A.I. Sideri, V. Tsioli, G.C. Fthenakis, A. Tzora, I. Skoufos, L.R. Quinlan, G. O'Laighin, A.M. Mullen, J.L. Kelly, S. Kearns, M. Biggs, A. Pandit, D.I. Zeugolis. Adv. Drug Deliv. Rev. 84 (2014) 257-277.

118. R.J. Egli, R. Luginbuehl. Swiss Med. Wkly. 142 (2012) w13647.

119. T.K. Hiong Teh, J.C. Hong Goh, S.L. Toh. Curr. Pharm. Des. 21(15) (2015) 1991-2005.

120. S. Sahoo, S.L. Toh, J.C. Goh. Biomaterials 31 (2010) 2990-2998.

121. E. Naghashzargar, S. Farè, V. Catto, S. Bertoldi, D. Semnani, S. Karbasi, M.C. Tanzi. J. Appl. Biomater. Funct. Mater. 13(2) (2014).

122. C. Yang, G. Deng, W. Chen, X. Ye, X. Mo. Colloids Surf. B Biointerfaces 122 (2014) 270-276.

123. C.N. Manning, A.G. Schwartz, W. Liu, J. Xie, N. Havlioglu, S.E. Sakiyama-Elbert, M.J. Silva, Y. Xia, R.H. Gelberman, S. Thomopoulos. Acta Biomater. 9 (2013) 6905-6914

124. Z. Yin, T.M.H. Nguyen, X. Chen, H.W. Ouyang. $13^{\text {th }}$ International Conference on Biomedical Engineering IFMBE Proceedings Vol. 23, 2009, pp. 1397-1400.

125. K.L. Moffat, A.S. Kwei, J.P. Spalazzi, S.B. Doty, W.N. Levine, H.H. Lu. Tissue Eng. Part A 15 (2009) 115-126.

126. A.P. Mathew, K. Oksman, D. Pierron, M.F. Harmand. Macromol. Biosci. 13 (2013) 289-298.

127. D.C. Surrao, J.W. Hayami, S.D. Waldman, B.G. Amsden. Biomacromolecules 11 (2010) 3624-3629.

128. S. Sahoo, L.T. Ang, J. Cho-Hong Goh, S.L. Toh. Differentiation 79 (2010) 102-110.

129. X. Cheng, C. Tsao, V.L. Sylvia, D. Cornet, D.P. Nicolella, T.L. Bredbenner, R.J. Christy. Acta Biomater. 10 (2014) 1360-1369.

130. S. Liu, M. Qin, C. Hu, F. Wu, W. Cui, T. Jin, C. Fan. Biomaterials 34 (2013) 4690-4701.

131. K. Karthikeyan, S. Sekar, M.P. Devi, S. Inbasekaran, C.H. Lakshminarasaiah, T.P. Sastry. J. Mater. Sci. Mater. Med. 22 (2011) 2721-2726.

132. Y. Zhou, L. Zhang, W. Zhao, Y. Wu, C. Zhu, Y. Yang. Biomaterials 34(33) (2013) 8269-8278.

133. A. Suwalski, H. Dabboue, A. Delalande, S.F. Bensamoun, F. Canon, P. Midoux, G. Saillant, D. Klatzmann, J.P. Salvetat, C. Pichon. Biomaterials 31 (2010) 5237-5245.

134. P.V. Kolluru, J. Lipner, W. Liu, Y. Xia, S. Thomopoulos, G.M. Genin, I. Chasiotis. Acta Biomater. 9 (2013) 9442-9450.

135. W. Liu, Y.C. Yeh, J. Lipner, J. Xie, H.W. Sung, S. Thomopoulos, Y. Xia. Langmuir 27 (2011) 9088-9093.

136. J. Lipner, W. Liu, Y. Liu, J. Boyle, G.M. Genin, Y. Xia, S. Thomopoulos. J. Mech. Behav. Biomed. Mater. 40 (2014) 59-68.

137. J. Xie, B. Ma, P.L. Michael, F.D. Shuler. Macromol. Biosci. 12 (2012) 1336-4. 
Chapter 15 